\title{
Measuring Consultant Radiologist workload: method and results from a national survey
}

\author{
Adrian P. Brady \\ Received: 4 February 2011 /Revised: 29 March 2011 / Accepted: 4 April 2011 /Published online: 21 April 2011 \\ (C) European Society of Radiology 2011
}

\begin{abstract}
Objectives The role of the Consultant Radiologist has changed substantially in recent decades, yet manpower planning is often based on older inappropriate methods of measuring Radiologist workload. We report a nationwide survey of Consultant Radiologist workload in Ireland in 2009.

Methods Relative value units (RVUs) were assigned to easily countable studies. Hospitals' activity was collated for the full calendar year of 2009. Radiologist time engaged in activities not easily counted (interventional and procedural work, multi-disciplinary meetings, teaching, administration, etc.) was separately measured.

Results Data were obtained from 28 of 38 public hospital radiology departments. Mean Consultant Radiologist workload across all hospitals was 57,659.1 crude RVUs/WTE and 103,987 net RVUs/WTE. A mean of $32.47 \%$ of WTEs are engaged in non-countable activity. Means of $85.35 \%$ and $65.73 \%$ of the required numbers of WTEs were available in 2009 to achieve respectively annual crude and net RVU/WTE figures of 45,000. Excluding Specialist Centres, plain films accounted for $28-41 \%$ of recorded activity, mammography for $0.8-5.8 \%$, US for $16-20 \%$, CT for $27-32 \%$ and MR for $5.9-15.8 \%$.

Conclusions Irish Consultant Radiologist staffing levels are well below appropriate international benchmarks for the current workload. Approximately one-third of radiologist time is engaged in activity not easily counted in study numbers.
\end{abstract}

Keywords Radiology · Health Manpower - Capacity building $\cdot$ Health Resources $\cdot$ Delivery of Health Care

\footnotetext{
A. P. Brady $(\bowtie)$

Faculty of Radiologists, Royal College of Surgeons in Ireland, Dublin 2, Ireland

e-mail: abrady@muh.ie
}

\section{Introduction}

The role of the Consultant Radiologist has changed substantially in recent decades, from that of a doctor whose primary activity was reporting plain film and crosssectional imaging studies, often in relative isolation from other clinical hospital services, to one where the radiologist is centrally involved in patient management through multidisciplinary teams, frequently with a significant direct therapeutic interventional role. Despite this evolution of radiolgists' centrality in patient care, manpower planning in radiology has tended to rely on out-dated methods of workload measurement, often based on crude measures of numbers of studies reported per Consultant.

In many jurisdictions, radiologist numbers are determined by local need, decided by local radiologist groups or hospitals. Thus, in most North American departments, if a department judges that additional radiologists are required to cope with workload, additional radiologists can be hired. However, in some jurisdictions, notably the UK and Ireland, Consultant Radiologist numbers in the public hospital service are centrally controlled, usually by governmental agencies. Thus, the process by which an over-worked radiology department can recruit additional radiologists is often tortuous, opaque and constrained by budgetary issues, which have no direct relationship to the workload demands on the department [1].

In 2009, the Faculty of Radiologists of The Royal College of Surgeons in Ireland (RCSI) studied the various methods available for calculating radiologist workload, and the uses to which the data obtained was being applied. The resulting Faculty report stated that:

"The use of crude study numbers to determine Radiologist workload and throughput is an oldfashioned, discredited and inappropriate misuse of data. Although the introduction of PACS/RIS technology in many Radiology departments makes it 
possible to acquire this data, it should not be used in an unfiltered and un-weighted manner....

Any measurement system used to assess workload and throughput needs to take account of the many variables... which influence how a Radiologist and a Radiology department works. The complexity of Radiologist work has increased very significantly in recent years... There is no universally applicable and universally accepted weighting system presently in use. Most weighting systems that exist at present were developed as tools to aid insurance reimbursement or other matters not directly concerned with Radiologist numbers. Efforts to assess workload and efficiency in individual departments must take account of local circumstances and clinical demand. A teaching hospital department, with many multi-disciplinary meetings per week, and a high complexity of work, cannot be expected to report as many studies per individual as a department which has fewer such commitments and less complexity of work.

If employing authorities... wish to acquire meaningful information on Radiology department workload or individual Radiologist workload, this should be done through the medium of an agreed, robust system of measuring the relative values of different studies, procedures and activities, which is adaptable to new professional and technical developments in the future".

Following this 2009 report, the Faculty proceeded with this survey, with the aspirations of establishing a standard, reproducible and adaptable method of collating the needed data, obtaining an accurate picture of current radiologist activity in Ireland, and providing the information necessary to plan workforce requirements at present and in the future.

Methods of recruiting radiologists and of determining the numbers of radiologists required vary across European countries, and across types of radiology departments within countries. Regardless of the health care system and methods of determining necessary radiologist numbers, we felt that the exercise described in this paper would be of interest to radiologists in any health care system, in Europe and elsewhere, as a reproducible method of determining Radiologist workload, validated by almost nationwide data from the public Irish hospital system.

\section{Materials and methods}

Method of measuring activity

The Pitman/Jones model of relative value measurement was published in Australasian Radiology in 2006 [2] on behalf of the Royal Australian and New Zealand College of Radiologists (RANZCR). The Australian relative value unit (RVU) system was adopted for this method, on the basis that it clearly separated radiologist, technologist and examination room utilisation costs, and that the radiologist cost is time-based. The general principle of the model is that "complex, large data-volume examinations with multiple images take a longer time to report, and consume more mental effort than studies with only a handful of images, such as a CXR. Another cardinal feature...is that the value of an examination depends on the number of regions covered; this is particularly so for CT, where a 'chest/ abdomen/pelvis' clearly takes more time and effort than a "chest", [2]. Various simplifications were necessary to allow rapid calculation of a valid workload measurement across departments, for example grouping together some studies of differing complexity in one RVU category. The Faculty of Radiologists, RCSI, in turn adopted the Pitman/ Jones model for our national survey, with a few slight modifications (assigning RVUs to intravenous urography studies and adjusting the definitions of categories of noncountable activity to reflect typical Irish radiology practices).

\section{Data collection}

A standard survey spreadsheet (with embedded calculations) and an explanatory document were sent to radiologists in 38 public hospital radiology departments in Ireland in May 2010, representing all public general hospitals and most public specialist hospitals (see Table 1).

An example of the spreadsheet used for data returns and calculations is shown in Table 2, with figures for a notional hospital included for demonstration purposes.

Table 1 Returns requested and received, by hospital type

\begin{tabular}{|c|c|c|c|}
\hline Hospital type & & $\begin{array}{l}\text { Returns } \\
\text { requested }\end{array}$ & $\begin{array}{l}\text { Returns } \\
\text { received }\end{array}$ \\
\hline $\begin{array}{l}\text { University teaching hospitals } \\
\text { with postgraduate radiology } \\
\text { trainees }\end{array}$ & & 8 & 8 \\
\hline $\begin{array}{l}\text { University teaching hospitals } \\
\text { without postgraduate } \\
\text { radiology trainees }\end{array}$ & & 6 & 4 \\
\hline County hospitals & & 17 & 11 \\
\hline \multicolumn{4}{|l|}{ Specialist hospitals } \\
\hline & Orthopaedic & 1 & 1 \\
\hline & Paediatric & 2 & 2 \\
\hline & Maternity & 3 & 1 \\
\hline & $\begin{array}{l}\text { Organ-specific } \\
\text { centre }\end{array}$ & 1 & 1 \\
\hline Total & & 38 & 28 \\
\hline
\end{tabular}


Table 2 Spreadsheet used for data returns and calculations, with example statistics

\section{Radiology Department workload statistics}

Name of hospital: Example

Year: 2009

1. Measurable reporting activity

Total number of studies

Relative

values
Total Relative value units (RVUs)

Plain films

Extremity

Spine

10600

1968

Chest or abdomen

25045

Skeletal survey

IVU

Mammogaphy

CT

Brain

Thorax (incl. Thorax \& upper abdomen)

Neck

Abdomen \& pelvis

Chest, abdomen \& pelvis

\section{MRI}

(incl. Brain, spine, MSK, body, angio, cardiac etc.)

3882

Grand Total RVUs

2. Radiologist time commitment other than in reporting activity (hours)

\begin{tabular}{lr} 
& \multicolumn{2}{c}{$\begin{array}{c}\text { Interventional/procedur } \\
\text { al/ Nuc Med activity }\end{array}$} \\
Monday & 8 \\
Tuesday & 7 \\
Wednesday & 9 \\
Thursday & 6 \\
Friday & 8 \\
Total hours & 38
\end{tabular}

Formal
teaching
(tutorials)

Multidisciplinary team
meeting (MDT) - actual
meeting conduct by lead

MDT

preparation

by

radiologist

Formal

administrative

work (meetings etc.)

15900

4920

37567.5

145

3505

4180

8350

1645

14120

2100

24960

46305

77640

284657.5

1

Grand total hours

87

3. Consultant radiologist numbers in department (whole time equivalents - WTEs)

Permanent

Temporary

Total
6

0

6

4. WTE Consultant radiologists occupied in non-reporting activity 2.351351351

5. Net WTE Consultant Radiologists available for reporting activity 3.648648649

\section{Crude reporting RVU per Consultant Radiologists FTE} 47442.91667

\section{Net reporting RVU per Consultant Radiologist FTE}


In Sect. 1 of the spreadsheet, each hospital was asked to record the total number (for the most recent year for which complete statistics were available) of studies in a variety of categories [plain films, mammography, ultrasound (US), computed tomography (CT) and magnetic resonance imaging (MRI)]; see Table 3. If studies were not specifically listed, respondents were asked to include their numbers in the nearest appropriate category (e.g. skull films were included in spine numbers). Since the majority of plain films fall into the chest, abdomen, spine or extremity groups, the lack of specific categories for other plain film studies was felt not to be likely to substantially skew the final numbers.

The RVU for a CT of chest/abdomen/pelvis was 27; the RVU for a CT thorax was 10, and for a CT of abdomen/ pelvis was 13. This apparent anomaly resulted from a quirk of the Australian reimbursement system, which does not differentiate between $\mathrm{CT}$ of chest/abdomen/pelvis and CT of neck/chest/abdomen/pelvis. Given that most of these studies relate to oncology patients, and thus frequently include CT of the neck in addition to the chest/abdomen/ pelvis, the RVU for the longer study was set at 27 , rather than the sum of CT chest and CT abdomen/pelvis (23) [Pitman A, personal communication]. To maintain consistency with already-published data from Australia, and because the same issue would apply to body CT in Ireland, these RVU values were retained for our survey.

Activity was recorded for work performed in public hospital departments only, or as part of fulfillment of Consultants' public hospital employment contracts; thus, in

Table 3 RVU levels assigned to studies in Sect. 1 data collection

\begin{tabular}{lll}
\hline Study type & & $\begin{array}{l}\text { Relative } \\
\text { value units }\end{array}$ \\
\hline Plain films & Extremity & 1.5 \\
& Spine & 2.5 \\
& Chest or abdomen & 1.5 \\
& Skeletal survey & 5 \\
& IVU (intravenous urography) & 5 \\
Mammography & & 5 \\
Ultrasound & Including abdomen, urinary, pelvis, & 5 \\
& breast, MSK, small parts, vascular, & \\
CT & other & 5 \\
& Brain & 7 \\
& Spine & 10 \\
& Thorax (including thorax and upper & 10 \\
& abdomen) & 10 \\
& Neck & 13 \\
& Abdomen and pelvis & 27 \\
& Chest, abdomen and pelvis & 20 \\
MRI & Including brain, spine, MSK, body, \\
& angio, cardiac, etc. & \\
\hline
\end{tabular}

those departments where contracts existed between employers and private operators for provision of services (particularly MRI), only those studies reported by consultants for which they did not receive private remuneration (i.e. public patients) were included in the data analysis. Any separately remunerated private practice work outside the public hospital department was not included in the analysis.

In Sect. 2 of the spreadsheet, reporting radiologists were asked to record the number of Consultant Radiologist hours per week devoted in their department to a variety of activities not amenable to easy case-by-case counting - listed in Table 4 . For procedural work, the radiologist time taken for procedures was considered the most accurate measure of procedural workload. This could be recorded on a per-procedure basis (involving onerous log-keeping) or on a per-session basis (e.g. Dr. X performing angiography between 08.30 and $12.30 \mathrm{~h}$ on Thursday implies $4 \mathrm{~h}$ of radiologist time per week spent on angiography, for the purposes of workload assessment). Time spent on associated direct patient care duties (especially clinical review pre- and post-intervention, patient counseling sessions and family meetings) was similarly logged as an integral part of Interventional Radiology. Procedures so accounted for were not included in RVU-based estimation of reporting workload. Time spent by Specialist Registrar trainees (SpRs) without direct Consultant participation was not included - this was an exercise in measuring Consultant workload only. As there are no validated RVU assignments available at present for Nuclear Medicine studies (including PET), the time spent in such work was also logged under the interventional/procedural/nuclear medicine category.

The same time-based method was used for preparation and conduct of multi-disciplinary team meetings (MDMs). While it is recognised that many MDMs are attended by more than one Consultant Radiologist, the time logged for preparation (column E) and conduct (column D) of the meeting was only logged by the lead radiologist who had primary responsibility for that particular MDM. Where radiologists spent appreciable time in extemporaneous film reviews for clinical colleagues coming to the department specifically for the purpose of second opinion, time on such informal clinico-radiological review meetings was similarly logged in the same category.

Table 4 Categories of non-countable (Sect. 2) activity recorded

Categories of non-countable radiologist activity recorded (by hours committed per week)

Interventional/procedural/Nuclear Medicine activity

Formal teaching (tutorials)

Multidisciplinary team meeting (MDT) conduct by lead radiologist

MDT meeting preparation by lead radiologist

Formal administrative work (meetings, etc.) 
Teaching time commitments was only logged for formal tutorials. Informal time teaching (e.g. while reporting CTs with a trainee) was not logged; this activity was captured in the CT RVU calculation.

Administration time was logged for the amount of time during working days spent by Consultants in activity directly relating to radiology management or administration work (e.g. department head activity, involvement in management committees, etc.).

This method is based on the total number of Consultant Radiologist staff positions in a teaching department and does not take into account the number of trainees in a department [2].

The number of whole-time equivalent Consultant Radiologists (WTEs) required to service this accumulated hourly need (calculated on the basis of the standard contractual commitment of a $37-\mathrm{h}$ week-allowance was not made for the small number of Consultant Radiologists on contracts specifying different hourly commitments) was then subtracted from the total number of WTEs available for work within the department (e.g. a Consultant whose commitment to the department was 2 days per week was listed as 0.4 WTE), leaving the number of WTEs available to service the counted study numbers in Sect. 1 . Whether or not an individual Consultant participated in the on-call rota was not relevant to this calculation (virtually all Consultants in public hospital departments share on-call commitments). The original Australian model, from which this calculation method was derived, was based on a $40 \mathrm{~h} /$ week commitment per WTE [2].

Division of the total counted RVU numbers from Sect. 1 by the total number of available WTEs gave the crude RVU/ Consultant WTE measurement. This measure takes no account of Sect. 2 activity (all interventional and other procedural work, nuclear medicine, formal teaching and administrative work, and preparation and conduct of MDMs). A more accurate measure of workload was calculated by dividing the total number of RVUs from Sect. 1 by the net available WTEs, after subtraction of those required to service Sect. 2 activity, the net RVU/Consultant WTE measurement.

2006 and 2009 RANZCR recommendations The 2006 Australian survey recommended 40,000 crude RVU/Consultant WTE per annum as an appropriate benchmark for a teaching radiology department. This recommendation took no account of procedural work and other non-countable activity, which would have been part of Net RVU/ Consultant WTE calculations [2]. In 2009, the RANZCR applied the same methodology to measuring activity in a larger and broader sample of Australian teaching hospitals, measuring activity from 2004 to 2006; they found that the mean activity level in the hospitals sampled had risen to 45,000 crude RVU/Consultant WTE per annum [3].

\section{Results}

Completed returns were received from 28 radiology departments (see Table 1). In all cases where complete annual returns were made, activity figures for the year 2009 were submitted; thus the data used in this survey (completed during 2010) represent the most recent full calendar year.

The results are summarised in Table 5, showing means and ranges for different hospital categories for the following measurements:

\section{Total RVUs}

Total WTE consultant radiologists

Crude and net RVUs per WTE

$\%$ of WTEs engaged in non-countable activity

Proportions of different types of non-countable activity $\%$ of WTEs required to achieve 45,000 crude and net RVUs/WTE actually in post

Results were grouped for different hospital types in an attempt to identify trends reflecting any possible differences in practice type and complexity. University teaching hospitals [most, but not all, of which have on-site Radiology Specialist Registrar trainees (SpRs)], in general undertake more complex tertiary-referral type work, in addition to providing services at a secondary-referral level to their relevant catchment areas. County hospitals, in general, serve a more localised population; while a higher proportion of their work might be expected to be secondary referral in type than in university teaching hospitals, some tertiary referral work is also performed in these institutions. Returns were also received from some specialist referral centres (one elective orthopaedic hospital, one maternity hospital neonatology radiology service, two university paediatric hospitals and one breast screening service); the data from these centres have been included, but are less applicable to general conclusions because of the very specific nature of the services provided in these centres.

The total number of WTE consultant radiologists available in the radiology departments surveyed ranged from 0.54 to 13 . University hospitals had larger departments, with greater numbers of WTEs and higher total RVU counts. County hospitals were staffed by between 1 and 5.1 WTE radiologists, dealing with total RVU activity levels between 63,278 and 317,260 per annum.

The crude RVU/WTE number was remarkably consistent across all hospital types, ranging from 48,873 in university teaching hospitals without trainees to 58,788 in county hospitals (and 62,025 in specialist centres). Net RVU/WTE numbers were more variable, ranging from 63,414 in university teaching hospitals without trainees to 126,376 in university teaching hospitals with trainees (and 155,296 in specialist centres); this in part reflects differences in complexity of work between different hospital groups, but 


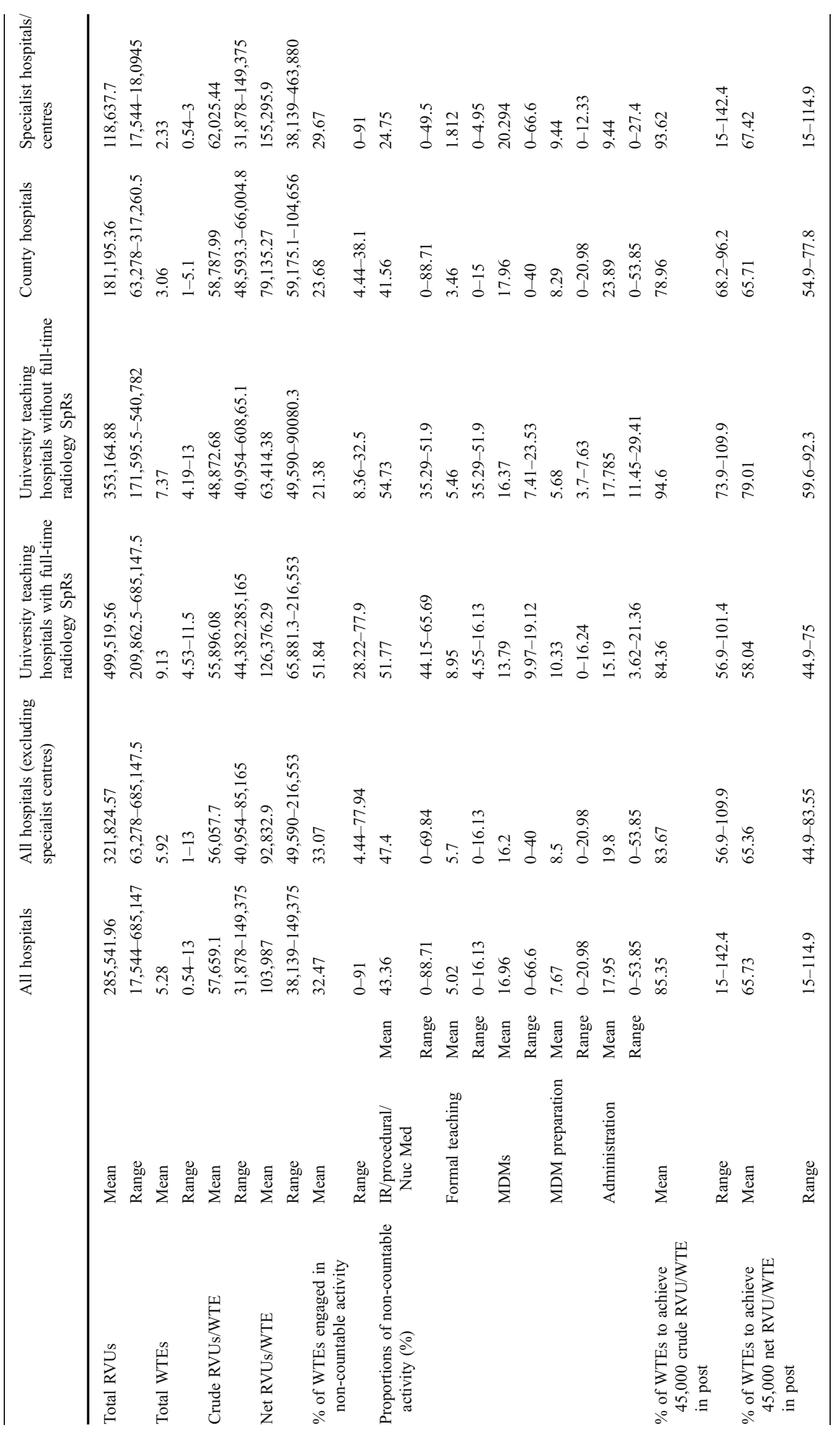


may also indicate differences in complexity within individual hospital groupings and differences in recording noncountable time commitments between hospitals (a possible bias in the reporting of this type of activity).

A mean of $32.47 \%$ (range $0-91$ ) of consultant radiologist WTEs were engaged in non-countable activity (recorded in Sect. 2 of the spreadsheet) across all hospitals. The commitment of this type of activity was greater (mean $51.84 \%$ ) in university teaching hospitals with full-time radiology SpRs. This might have been expected, given that this group accounts for a majority of the larger tertiaryreferral hospitals in the country, with more complex interventional workloads, more teaching commitments and more MDMs than some other groups.

Figure 1 shows the proportions of each type of noncountable activity by hospital group. Interventional, procedural and nuclear medicine activity accounts for over $40 \%$ of this activity, in all hospital types except specialist centres (because of sample size and variability in methods of data inclusion and return, the data from Specialist Centres are of limited value).

With respect to the numbers of consultant WTEs required to achieve 45,000 crude and net RVUs per WTE (Table 5), among the hospitals sampled, 32.47 additional WTEs would have been required in 2009 to achieve the crude benchmark of 45,000 RVUs/WTE; 90.71 additional WTEs would have been needed to achieve the net benchmark of 45,000 RVUs/WTE. A mean of $85.35 \%$ of the required numbers of WTEs was available in 2009 to achieve a crude RVU/WTE figure of 45,000; a mean of $65.73 \%$ of the required numbers were available to achieve 45,000 net RVUs/WTE.

Table 6 and Fig. 2 group the relative proportions of different study types for each category of reporting hospital.
Excluding specialist centres, plain films accounted for 28 $41 \%$ of recorded activity (measured as a mean for each group), mammography for $0.8-5.8 \%$ (many hospitals do not have any mammography service), US for $16-20 \%$, CT for $27-32 \%$ and MR for $5.9-15.8 \%$ (many hospitals access MRI through privately funded arrangements; this MR activity is not included in the survey, and thus the MR proportion recorded underrepresents the actual impact of MR, public and private, on workload).

Ten hospitals from which data were requested were unable to make returns. In two cases, this was because of declared unhappiness with the process being used, including feelings that the methodology did not make sufficient allowance for variables such as the presence or absence of radiology SpRs within a department and the presence of Consultant Radiologists holding different contract categories within a single department. One hospital formally stated they would not be able to provide data because their Radiology Information System (RIS) could not produce accurate or reliable data. One hospital made no return because of an absence of radiologist staff to collate information (during a time of staff transition). One specialist centre opted not to make a return, as it was felt its input would not be germane to the main thrust of the survey. No explanation was received for the lack of returns from five county hospitals.

\section{Discussion}

Measurements used elsewhere and in the past

Historically, the number of studies reported by individual radiologists was used as a crude measure of radiologist activity.
Fig. 1 Mean proportions of non-countable activity

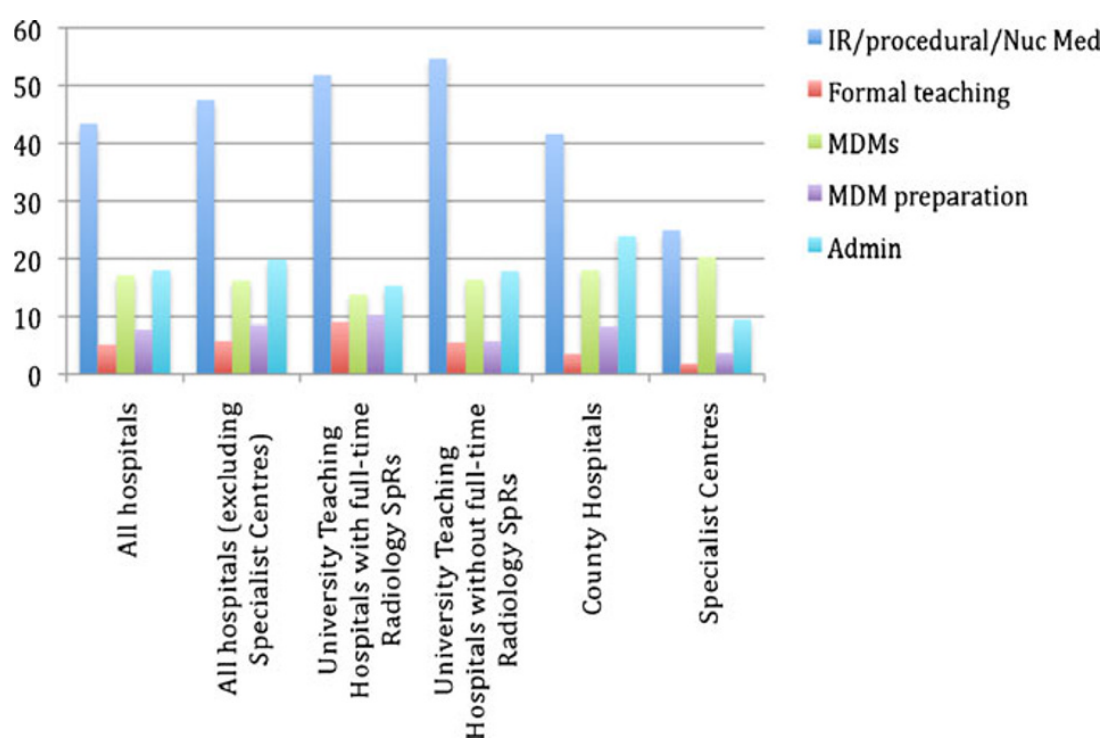


Table 6 Mean proportions of different study types

\begin{tabular}{|c|c|c|c|c|c|}
\hline & Plain films & Mammography & US & $\mathrm{CT}$ & MR \\
\hline All hospitals & 34.08 & 5.78 & 19.74 & 27.18 & 13.22 \\
\hline All hospitals (excluding specialist centres) & 35.34 & 2.91 & 18.57 & 32.49 & 10.68 \\
\hline University teaching hospitals with full-time radiology SpRs & 28.48 & 4.68 & 16.43 & 34.54 & 15.86 \\
\hline University teaching hospitals without full-time radiology SpRs & 33.34 & 5.21 & 17.17 & 30.82 & 13.46 \\
\hline County hospitals & 41.06 & 0.79 & 20.64 & 31.61 & 5.89 \\
\hline Specialist centres & 28.24 & 18.99 & 25.1 & 2.75 & 24.92 \\
\hline
\end{tabular}

1. During the 1980s and 1990s, many Irish hospitals utilised an Irish-developed proprietary RIS, which assigned work units to different radiologic studies. The figures used were often erroneously utilised to assess radiologist activity. In fact, the work unit allocations were originally intended to reflect radiographer workload and were heavily weighted to reflect the hospital department in which the system was first developed. Thus, work unit assignments existed in the system for procedures commonly performed in that department at the time of the system's development, while there were no assignments for procedures not performed at that particular site. In addition, an arbitrary maximum number of 60 work units was embedded in the system; thus, even if a department tried to keep the work unit assignments up to date, they had no facility to assign an appropriate value to a new, complex and very timeconsuming interventional procedure or crosssectional imaging study that had not been available when the system was first developed.
2. Comhairle na nOspidéal, the Irish statutory body that had responsibility for considering and approving applications for Consultant posts until 2009, used a modification of the Foresterhill system of workload assessment. This system was developed in Aberdeen and was used in Scotland until the 1990s. A time of 3 min was allocated to a CXR report, and other categories of radiologist work were allocated multiples of the time allocated to a CXR. Comhairle used three classes of studies in calculating radiologist workload:

Class 1: Plain films

1 point

Class 2: Barium studies, US, IVUs, tomograms, etc. $\quad 7$ points

Class 3: CT, nuclear medicine, angio/IR, MRI

24 points

The total points of the workload in a department (as submitted by the hospital in an application for a radiologist post) were added, and an additional 10\% weighting was added for teaching departments. The total points score was then divided by the number of
Fig. 2 Mean proportions of different study types

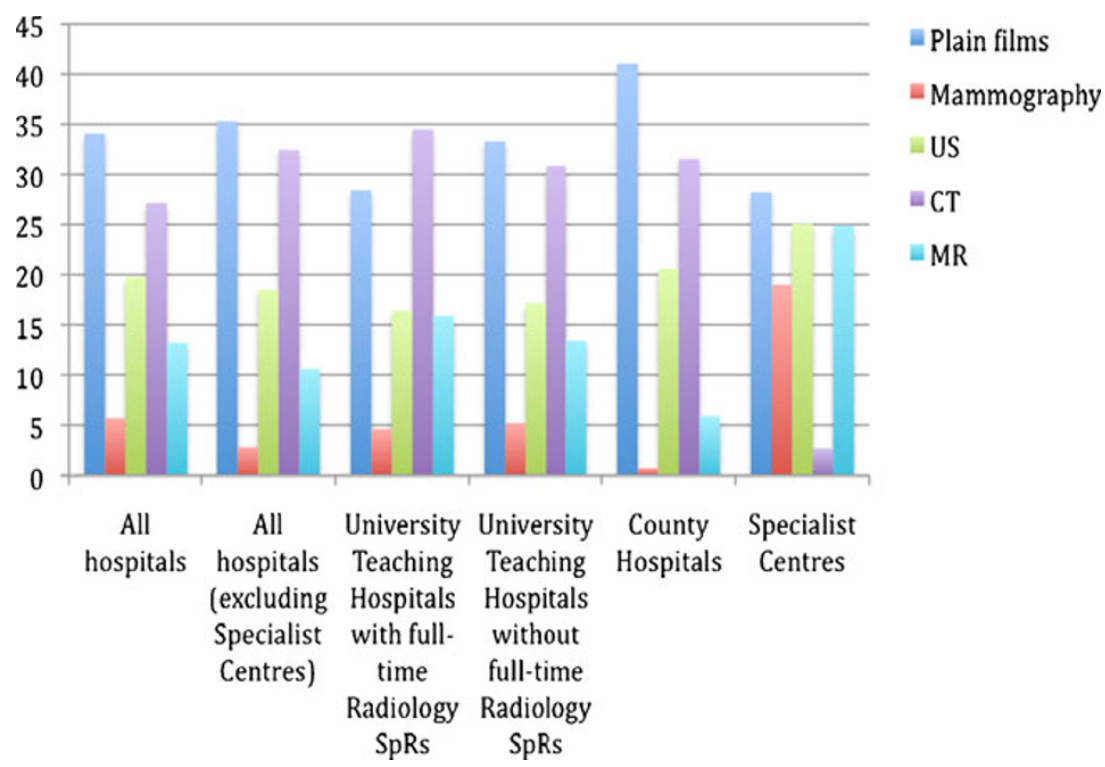


sessions (based on 11 clinical sessions per consultant and 46 working weeks per year), to give the points per session (a session was defined as a 3-h period in the contract in use at that time). Comhairle did not have an absolute figure forming the basis of approval for posts, but sought to achieve an average of 100 points/session for larger hospitals and 65 points per session for smaller hospitals.

3. In the USA, published surveys showed that in 20062007, an average of 14,900 examinations were reported per full-time radiologist per annum, with primarily academic groups performing about one-third fewer studies than others [4]. It should be noted that these published surveys were not in any way intended to act as a template to mandate the number of radiologists required in any department to cover the workload of that department. They simply represented a collation of data indicating the position on the ground; radiologist numbers are not centrally controlled in North American departments, and additional radiologists are recruited by hospitals or practices according to need, determined locally.

4. Relative value units (RVUs) are a measure of activity utilised in a number of countries, including the USA, Canada, Australia and New Zealand. A variety of RVU systems are used in different countries, some of which measure both professional and technical components of workload, and some of which can be utilised more accurately to determine the specific radiologist component to workload, on a time basis. RVUs are designed to reflect the time required for a procedure and the complexity and/or intensity of the work, but are primarily a tool to determine reimbursement for work done, rather than to measure individual workload [2]. Various models of measuring radiologist workload were considered by Pitman and Jones in developing the RANZCR system, including crude study numbers, the Ontario reimbursement system and the US RBRVS [Resource-Based Relative Value Scale, used by Medicare and most Health Maintenance Organisations (HMOs) in the US]. Procedures are assigned a relative value, adjusted by geographic region. Prices are determined based on three separate factors: physician work (52\%), practice expense (44\%) and malpractice expense (4\%)] [2].

5. In 1990, The Royal College of Radiologists (UK) (RCR) made recommendations on radiologist numbers based on the total number of studies performed and reported in radiology departments $(12,500$ examinations per annum for a District General Hospital Consultant Radiologist). While it was recognised that individual radiologists would not all necessarily report equal numbers of studies (because of different subspe- cialty interests, varying non-service commitments, differing modality commitments, etc.), it was felt that across radiology departments, workload could be evened out reasonably in this way.

The RCR document on Workload and Manpower in Clinical Radiology produced in 1999 (superseding the 1990 document) recognised that this old-fashioned method of calculating workload was no longer appropriate [5]. The 1999 document's manpower recommendations were based on suggested levels of appropriate workload in a notional half-day, broken down according to whether the work involved general (plain film) radiology reporting, general ultrasound, barium studies, $\mathrm{CT}$, MRI or vascular and interventional radiology. For example, three vascular/IR cases was considered an appropriate consultant workload in a notional half day $(3.5 \mathrm{~h})$, while 70 general reporting cases was the suggested workload in the same time period. These recommendations were withdrawn in 2006. In 2008, the RCR issued their latest guidelines on workforce planning; they no longer utilise specific study number recommendations, taking account of the changing and increasing role of radiology in clinical management, and the varying complexity of radiologic tasks undertaken by consultant radiologists [6].

The 2008 RCR document summarises the role of the radiologist as follows:

- "Direct image acquisition (e.g. ultrasound, fluoroscopy) and image-guided therapy (vascular and non-vascular intervention)

- Reporting of images acquired by others (e.g. CT, MRI, plain films)

- Consultation and discussion with other clinicians, through one-to-one consultations, multidisciplinary meetings or other means of communication

- Audit, management, supervision, teaching and research

The balance of these activities, clinical and nonclinical, will vary greatly from individual to individual.

Many factors influence throughput in the different components of the radiologist's role:

\section{Image acquisition and image-guided therapy}

- The time required for interaction with the patient as well as the technical performance of the procedure

- The complexity of the cases and procedures

- The need to provide access to procedures on an emergency basis in addition to elective booked cases

- The time required for pre-procedural patient assessment and consent, and post-procedural patient 
follow-up (this is an increasing feature of interventional work)

\section{Reporting}

- The complexity of the studies being reported

- The need for comparison with previous studies and other modalities, and the ease with which these can be accessed in a timely fashion

- The reporting technology available, including transcription and report authorisation methodologies

- The frequency and likelihood of interruption while reporting

- The administrative and secretarial support available

- The availability and efficiency of systems for communicating urgent reports and findings to referrers

- Commitments to teaching and supervision

- Consultation

- Clinico-radiologic meetings, including the time required to prepare cases for these meetings

- Face-to-face consultations with clinicians (planned or ad-hoc)

- Telephone discussions

- Vetting of requests to determine protocols and appropriateness

- Written and e-mailed communication with other clinicians

Non-clinical duties

- Audit

- Participation in departmental, hospital or wider management

- Participation in education provision and support

- Teaching and examining medical students, nursing and paramedical staff, specialist registrars and other junior doctors

- Supervision of specialist registrars

- Participation in RCR or Faculty of Radiologists activity

- Research" [6]

6. Weighting systems have been devised in some countries, which may be helpful in assessment of workload in departments with a high proportion of complex investigations and procedures. The British Society of Cardiovascular Imaging published a document in September 2008 on this issue, proposing relative scores for different plain film, CT and MR studies relevant to cardiac imaging [7].

7. A Japanese survey of radiologist supply and workload, published in 2008, reported that Japan had the lowest number of radiologists per million of population (36 per million) in 2004 among 26 Organization for Economic Cooperation and Development (OECD) countries. The UK figure was also 36/million, followed by Ireland at $45 /$ million, and increasing to $226 /$ million in Greece, with a mean of 101/million across all 26 countries [8].

Staffing implications of survey results

This nationwide survey of Consultant Radiologist workload in the public hospital system of the Republic of Ireland represents one of the first such efforts to accurately measure the work output and productivity of a major medical specialty across the entire public health care system of a country. While radiology is a specialty which lends itself to some extent to accurate quantification of workload, the reporting of non-countable (Sect. 2) activity indicates that this aspect of radiologists' work now accounts for, on average, $32.47 \%$ of radiologists' working time.

The mean crude RVU/WTE measurement of Irish radiologist workload is $57,659.1$ RVUs, substantially in excess of the documented Australian measurement of 40,000 RVUs reported in 2006, and the subsequent Australian measurement of 45,000 RVUs published in 2009. At the time the workload data were recorded, the hospitals making returns had a total of 147.88 WTE Consultant Radiologists in post. In order to achieve the 2009 Australian benchmark of 45,000 RVUs per Consultant Radiologist, an additional 32.47 WTE posts would have been needed in 2009 in the hospitals participating in this survey (Table 6). The number of WTEs required to achieve a Net RVU/WTE value of 45,000 RVUs is also listed in Table 6; the total in 2009 would have been 90.71 additional WTEs. These numbers are based upon the presumption that non-countable activity would not increase above present levels and that this activity would be shared among a greater number of radiologists. Taking into account the growing contribution of non-countable Sect. 2 activity, a more accurate staffing deficit in the participating hospitals is approximately 100 WTE posts.

Approximately $85-90 \%$ of the total public hospital Consultant Radiologist numbers in Ireland are employed in the hospitals that made returns to this survey; on that basis an additional 38 (crude RVUs) to 107 (net RVUs) WTEs would have been required countrywide in 2009.

Non-countable (Sect. 2) activity

A notable outcome of this survey is that it establishes (albeit on a self-reported basis only) a fact that has been anecdotally known to radiologists for some time: on average, $32.47 \%$ of Consultant Radiologists' working time is now taken up with meaningful work that cannot be counted in simple study numbers. Some aspects of this non- 
countable work have long been a feature of radiology: teaching and administrative duties have existed as long as our specialty. However, the most time-consuming elements of this non-countable activity represent relatively recent or continually developing aspects of our work; $67.99 \%$ of this activity is accounted for by interventional, procedural and nuclear medicine activity, and preparation for and conduct of MDMs.

The move towards a multi-disciplinary model of patient care, which is best-developed in, but not exclusive to cancer care, has rightly brought radiologists to a central role in medical and surgical teams. This is a positive strategy, in terms of making the best use of all relevant expertise and information in optimising patient outcomes. However, it has added significantly to radiologists' workload; on average, preparation for an hour-long MDM occupies at least another hour of a radiologist's time, a 1-h MDM thus involving at least $2 \mathrm{~h}$ for a consultant radiologist (similar comments are likely to apply to pathologists' MDM work; surgeons, physicians, oncologists, etc., rely more on their day-to-day contact with and knowledge of their patients during MDMs, and may not need to commit as much time to specific meeting preparation).

Interventional radiology (IR) continues to evolve as a sub-specialty; procedures offered as part of an IR service vary from hospital to hospital, according to local demand and expertise. Many procedures are now performed by interventional radiologists with the intention of treating conditions previously requiring open surgery, often requiring commitment of hours of a consultant radiologist's time per individual case. Furthermore, proper provision of an IR service requires more than simply the performance of procedures; pre- and post-procedural patient care is often properly the responsibility of the treating radiologist, and requires significant time commitment. As would be expected, the proportion of non-countable time devoted to IR activity varies across the different types of hospitals; however, in all groups, over $40 \%$ of total non-countable activity is accounted for by interventional, procedural and nuclear medicine activity (nuclear medicine comprising the smallest proportion).

\section{Evolving radiology activity}

Even in those studies that lend themselves to relatively easy unit counting (plain films, ultrasound, CT, MRI), time commitments continue to evolve. The RVUs assigned to the different study groups in the Australian model used in this survey were based on an economic costing model of radiologist time devoted to these study types, relative to a standard of the time required to report an average chest Xray (CXR). These timing relativities were assigned in 2003. Since that time, new study types have been developed that may not easily be encompassed in the existing groups and sub-groups. For example, PET/CT interpretation is a more complex and time-consuming exercise than $\mathrm{CT}$ interpretation; PET/CT studies (or the time spent reporting them) should therefore be counted separately from conventional CT. Some MRI studies (e.g. breast MR, cardiac MR, MR spectroscopy) may require considerably more time than conventional single-level MR. Multidetector CT (MDCT) has largely replaced single-slice CT, thus increasing greatly the size of the average dataset to be reviewed for each CT study; this is compounded by the increasing use of multiphasic CT studies for tissue characterisation. Studies such as CT colonography and CT coronary angiography, which rely on large data sets, multiplanar reconstructions and sophisticated post-processing, are much more timeintensive for radiologists than conventional abdominopelvic CT. Thus, application of RVU measures to 2009 practices, based on 2003 time measurements, introduces another means of underestimation of the time involved in delivering modern radiology services. RVU assignments to specific study types may require periodic re-measurement to allow for these developments.

\section{Other issues}

- This exercise was not designed or intended to collect data in other categories collated by health care authorities, e.g. waiting times, report turn-around time, etc.

- Data collected related to all activity on public hospital sites, permitted by consultants' public hospital contracts, and did not differentiate between on-site public and private activity. Separately remunerated activity outside the public hospital radiology department was not included.

- The accuracy of the counted numbers of studies reported by participating radiology departments is likely to be subject to some variability. While study types have been "lumped" together as much as possible, in order to achieve relative uniformity for comparison purposes, the method by which studies are recorded in different statistical systems presently used is not uniform. Thus, for example, some older statistical systems may have recorded CTs of chest/abdomen/ pelvis as 3 separate studies, rather than a single study attracting 27 RVUs. MRI study counting in some older RIS systems may also be subject to some variability. Because of the variable nature of radiology data systems presently in use, it may not have been possible to counteract these inaccuracies entirely in recording study numbers, despite the guidelines given for data collation. This does not imply that the overall thrust of the statistics produced by this exercise is inaccurate. 
However, any comparisons between radiology departments needs to take account of any differences in the RIS systems they use and their ability to produce comparable data. In general terms, those departments relying on data produced by modern Picture Archiving and Communication Systems (PACS)/RIS systems are more likely to be reliably comparable than departments that use manual or out-moded RIS systems. The planned integration of all public hospital radiology departments in Ireland through the National Integrated Medical Imaging System (NIMIS) project (expected to be complete by the end of 2013) will ensure that statistics produced by departments for future comparison purposes will be of a more uniform nature.

- The introduction of PACS/RIS facilities to many Irish radiology departments has resulted in significant improvements in efficiency in terms of speedy delivery of study images and reports to referrers. The NIMIS project will consolidate these efficiencies. However, most such systems employ voice-recognition dictation facilities for report generation; this places the burden of report editing and correction (previously largely carried by transcriptionists, with final radiologist review) on the reporting radiologist, thereby increasing the time required to report each individual study by $20-30 \%$ relative to the time required in the older dictate-to-tape (with separate transcription) systems [9, 10]. This factor must be taken into account in establishing workload norms in the PACS era.

- The contribution of the presence of postgraduate medical trainees in diagnostic radiology (specialist registrars, SpRs) in a hospital radiology department was considered in assessing consultant radiologists' workload. A certain amount of the workload of a department with SpRs will be primarily undertaken by the trainees; this is appropriate, and the level of responsibility taken by SpRs is graded according to their experience, the amount of formal training they have completed and their individual performance. Thus, for example, much of the preliminary reporting of studies and some of the procedural work (including hands-on ultrasound scanning and some interventional radiology procedures) may be performed by $\mathrm{SpRs}$ in these departments. Furthermore, SpRs would frequently be the primary radiologists collating and preparing cases for multi-disciplinary meetings (MDMs), and would often also be the first point of contact for consultation on cases with other specialties, reducing the likelihood of interruptions to consultants engaged in service provision, and the amount of time consultant radiologists must devote to guiding and educating junior doctors in other specialties about the best use of radiology services. In most (but not all) Irish depart- ments with SpRs, on-call services are provided on a first-call basis by SpRs, with consultants second on-call. For all these reasons, it is sometimes argued that, in departments with radiology SpRs, much of the measured workload is not primarily undertaken by consultant radiologists, unlike in those departments without SpRs, where all radiology workload is primarily provided at the consultant level. In departments where on-call services are delivered primarily or exclusively by consultants, increasing on-call demands may place substantial strain on the ability of consultants to provide working-day and out-of-hours cover.

- However, counter-arguments exist to the contention that consultant workload is lower in training departments. The amount of work performed unsupervised by SpRs is variable, but generally small. Some plain film (especially emergency department) reporting is performed in many departments by SpRs, but the general practice is that trainees who have not passed the postgraduate specialty fellowship exam (Fellowship of the Faculty of Radiologists, Royal College of Surgeons in Ireland, FFRRCSI), which is initially taken usually after completion of approximately $70 \%$ of the minimum 5 years' formal specialty training in radiology, do not report other studies unsupervised. Thus, the bulk of work performed by $\mathrm{SpRs}$ would be individually checked by a consultant, this process often taking at least as long as its primary reporting, often longer. One published study of staff (consultant) radiologist output in an academic centre alone and while engaging in informal teaching of a radiology trainee showed a decrease in output of approximately $50 \%$ while engaging in teaching [11]. Furthermore, procedural work involving teaching a trainee is often slower than if the same procedure were performed by a consultant working alone. This can also apply to supervising work performed on call by a trainee. While having SpRs as the first contact for other specialties can reduce interruptions to consultant-delivered work, interruptions by the SpRs may negate any benefit. Finally, even if SpRs prepare and conduct MDMs, multi-disciplinary patient care requires the presence and input of fully trained consultants, which may involve as much of the consultant's time as if they primarily conducted the meeting themselves.

- Therefore, in the absence of any certainty as to whether having SpRs in a radiology department lessens or increases consultant workload, arguments can be made on either side. The presence of SpRs has not, therefore, been allowed for in calculations of consultant workload for the purposes of this study (this was also the case in the Australian published studies $[2,5])$. 
- The question of recording the time spent scanning patients in ultrasound by a consultant separately from the RVUs assigned to the ultrasound studies was considered; while we understood that a consultant scanning patients may spend more time in the process than one reporting on images acquired by sonographers, for the purposes of attempting to obtain data in a uniform manner across the country, we asked that the number of ultrasound studies simply be recorded under the appropriate RVU category. One paediatric hospital counted this activity as procedural time; however, in all other returns (in particular, in all general hospitals, which provided the most robust data), ultrasound scanning by consultants was not specifically allowed for. Scans were simply assigned the standard RVU per case, regardless of who did the actual scanning.

- Double-reading of studies, especially in mammography, is not allowed for in this measurement process (unless studies are counted twice, which was not done for the purposes of this survey); in effect, double-read studies require twice the time commitment actually recorded.

- A considerable component of workload in larger teaching hospitals, and especially in those with a significant cancer-care profile, involves reviewing studies performed in outside hospitals, referred in for second opinions or for multi-disciplinary care. Some, but not all of this activity has been captured in the time recorded for MDM preparation.

- Data recorded from the small number of specialist centres must be subject to interpretation in the light of their specific circumstances. Some of these centres share consultant staff with larger general hospitals, and much of the Sect. 2 non-countable activity that should be applied to these centres has been captured in the returns for their allied general hospitals. In the case of paediatric hospitals, radiology activity is often more time-consuming because of the nature of the patient population. Thus, most or all ultrasound scanning is directly performed by a consultant (often with uncooperative patients), taking relatively more time than in adults. This fact has been allowed for by inclusion of time spent scanning in the procedural time recorded for one paediatric hospital. For these and other reasons, the specialist centre activity returns can skew mean levels for general hospitals; as this survey was primarily intended to assess workload in general hospitals, representing the vast majority of radiology activity in Irish public hospitals, the specialist centre activity figures have not been included in the calculation of means in column 5 of Table 5, which therefore represents the most-robust and accurate indication of general radiology activity.
Other recognised shortcomings of the Pitman/Jones model included the following [5]:

- Australian RVUs do not reflect the other three components of radiologist work described by the American College of Radiology (ACR) [12]: inherent skill and technical proficiency, intensity and mental effort, medico-legal risk and stress. Nonetheless, this survey is, we believe, an accurate appraisal of Consultant Radiologist time commitment to work.

- The model does not capture reporting workload performed by trainees independently of consultants. As most SpR work in Ireland is supervised and separately verified by consultants, this is not likely to significantly bias results.

- The model does not capture work conducted by other staffing groups in radiology departments, e.g. radiographers, nurses and administrative staff. Again, this is not germane to the specific purpose of this survey, which relates to consultant radiologist activity.

- The model does not identify unreported studies (leakage) or unreported backlog - it assumes that all studies are reported. An Irish national survey of unreported studies published in December 2010 confirmed that, while small unreported backlogs had existed in 2008 and 2009, these had been entirely cleared by October 2010 [13].

- The RVU model only measures one aspect of one stage of the multistage imaging testing cycle (from request through study, report and communication of result).

Possible future directions

The methodology used in this survey is not perfect, but it represents the best available validated and published model for measuring consultant radiologist workload. Among the strengths of the template used are its ease of application to departments of varying size and offering services of varying complexity, and the fact that it includes a method of capturing the large amount of radiologist activity that is not easily counted in study numbers. One of its weaknesses is the reliance on selfreporting for this non-countable activity. We believe that the level of bias in recording of this Sect. 2 activity is minimal; the similarity in the amount of time and the proportion of overall time devoted to each type of noncountable activity across all hospital groups is striking. Nonetheless, in the interest of rigorous data collection, it would be best if future versions of this recording template could reduce the number of activities for which individual time recording is used. Ideally, future surveys should also assign RVUs for nuclear medicine (including PET) and, if possible, interventional cases. 
The RVU assignments used in this survey are, to some extent, already outdated. This applies particularly to evolving complex study types, particularly in CT and MR, which were not accounted for in the 2003 RVU tables (discussed above). Future workload surveys of this type will need to modify RVU assignments (and, if necessary, introduce new categories), allowing for evolving study complexity, to ensure accuracy and relevance of results.

Although the methods used to determine staffing levels in public health care systems vary significantly from country to country, The Faculty of Radiologists, RCSI, believes that our adaptation of the Australian model for workload calculation represents a useful tool applicable to any country's hospital system. Multi-national agencies, such as The European Society of Radiology (ESR), may find value in a reproducible system of measurement such as we describe, as a means of identifying patterns and trends in workloads across disparate healthcare systems in many countries.

A single survey such as this is an important exercise for establishing a snapshot of activity at a single point in time. Equally important is the need to follow up with repetitions of the same exercise in future years, to establish any trends in workload, and to gauge whether or not this first survey has led to action to alleviate excessive workload demands.

\section{Conclusions}

Maintenance of safe radiology services to patients cannot be achieved in the absence of adequate resources; expanding or new demands cannot be safely met simply by demanding more work from the existing staff. This survey shows that Irish consultant radiologist staffing levels are already well in excess of appropriate international benchmarks. Before more is asked of the existing radiologist complement, attention must be paid to bringing their available numbers up to internationally acceptable levels.

The data obtained in this survey are remarkably consistent across the hospitals sampled (representing the majority of public hospital departments in Ireland). Given this finding, the Faculty of Radiologists strongly recommends that the method of radiologist workload measurement used in this survey should form the basis for all future such measurement in Ireland, replacing other, less robust and less reproducible methods that have been used in the past. Employing authorities should formally adopt this method; future modifications of the method (incorporating evolving practices and radiology modalities) should be jointly undertaken as required by the Faculty and employing authorities, taking account of international developments in the field of radiologist workload measurement.
The method of measuring and calculating radiologist workload described in this paper is easily adapted for use in other jurisdictions. International societies such as the ESR should consider it as a possible template for use in crossborder comparisons.

Acknowledgements The author would like to acknowledge the support, advice and suggestions of the members of the Board of The Faculty of Radiologists, Royal College of Surgeons in Ireland, and the efforts of radiologist colleagues in the 28 departments who submitted data returns.

\section{References}

1. Tallaght Hospital Review (2010) Report of the Review of Radiology Reporting and the Management of GP Referral Letters at Adelaide and Meath Hospital (Dublin), incorporating the National Children's Hospital, (AMNCH) [Tallaght Hospital] September 2010. URL http://www.hse.ie/eng/services/ Publications/services/Hospitals/Tallaght $\% 20$ Hospital\%20Review $\% 202010$.pdf

2. Pitman AG, Jones DN (2006) Radiologist workloads in teaching hospital departments: Measuring the workload. Australas Radiol 50:12-20

3. Pitman AG, Jones DN, Stuart D, Lloydhope K, Mallitt K, O'Rourke P (2009) The Royal Australian and New Zealand College of Radiologists (RANZCR) relative value unit workload model, its limitations and the evolution to a safety, quality and performance framework. J Med Imaging Radiat Oncol 53:450 458

4. Bhargavan M, Kaye AH, Forman HP, Sunshine JH (2009) Workload of radiologists in United States in 2006-2007 and trends since 1991-1992. Radiology 252(2):458-67

5. Royal College of Radiologists (1999) Workload and manpower in clinical radiology. BFCR(99)5.

6. Royal College of Radiologists (2008) How many radiologists do we need? A guide to planning hospital radiology services. BFCR (08)17 . URL https://www.rcr.ac.uk/docs/radiology/pdf/BFCR(08) 17 Workforce.pdf

7. British Society of Cardiovascular Imaging (2008) Benchmarking in Cardiovascular Imaging (Revised 2009). URL http:// www.bsci.org.uk/downloads/cat_view/45-benchmarking? limit $=5 \&$ limitstart $=0$ \&order $=$ date $\& \overline{\text { dir }}=\mathrm{ASC}$

8. Nakajima Y, Yamada K, Imamura K, Kobayashi K (2008) Radiologist supply and workload: international comparison-Working Group of Japanese College of Radiology. Radiat Med 455-465.

9. Gale B, Safriel Y, Lukban A, Kalowitz J, Fleischer J, Gordon D (2001) Radiology report production times: voice recognition vs transcription. Radiol Manage 23:18-22

10. Glaser C, Trumm C, Nissen-Meyer S, Francke M, Küttner B, Reiser M (2005) Speech recognition: impact on workflow and report availability (article in German). Radiologe 45:735-742

11. Jamadar DA, Carlos R, Caoili EM, Pernicano PG, Jacobson JA, Patel S et al (2005) Estimating the effects of informal radiology resident teaching on radiologist productivity: what is the cost of teaching? Acad Radiol 12:123-128

12. Allen B Jr (2007) Valuing the professional work of diagnostic radiology services. J Am Coll Radiol 4:106-114

13. Health Service Executive Ireland (2010) Report of the HSE National Radiology Survey, December 2010. URL http:/www.hse.ie/eng/ services/Publications/services/Hospitals/radiosurvey.pdf 